TRANSACTIONS OF THE

AMERICAN MATHEMATICAL SOCIETY

Volume 359, Number 7, July 2007, Pages 3337-3355

S 0002-9947(07)04106-2

Article electronically published on February 13, 2007

\title{
DISKLIKENESS OF PLANAR SELF-AFFINE TILES
}

\author{
KING-SHUN LEUNG AND KA-SING LAU
}

\begin{abstract}
AвStract. We consider the disklikeness of the planar self-affine tile $T$ generated by an integral expanding matrix $A$ and a consecutive collinear digit set $\mathcal{D}=\{0, v, 2 v, \cdots,(|q|-1) v\} \subset \mathbb{Z}^{2}$. Let $f(x)=x^{2}+p x+q$ be the characteristic polynomial of $A$. We show that the tile $T$ is disklike if and only if $2|p| \leq|q+2|$. Moreover, $T$ is a hexagonal tile for all the cases except when $p=0$, in which case $T$ is a square tile. The proof depends on certain special devices to count the numbers of nodal points and neighbors of $T$ and a criterion of Bandt and Wang (2001) on disklikeness.
\end{abstract}

\section{INTRODUCTION}

Let $M_{n}(\mathbb{Z})$ denote the set of $n \times n$ matrices with entries in $\mathbb{Z}$ and let $A \in M_{n}(\mathbb{Z})$ be expanding, that is, all eigenvalues of $A$ have moduli $>1$. Suppose $\operatorname{det} A=q$. We let $\mathcal{D}=\left\{0, d_{1}, \cdots, d_{|q|-1}\right\} \subseteq \mathbb{Z}^{n}$ be a set of $|q|$ distinct vectors. We call $\mathcal{D}$ a digit set and $(A, \mathcal{D})$ a self-affine pair. Let $T=T(A, \mathcal{D})$ denote the set of radix expansions with respect to the pair $(A, \mathcal{D})$, i.e.,

$$
T=\left\{\sum_{k=1}^{\infty} A^{-k} d_{j_{k}}: d_{j_{k}} \in \mathcal{D}\right\}=\sum_{k=1}^{\infty} A^{-k} \mathcal{D} .
$$

It is easy to show that $T$ satisfies the set-valued functional equation $A T=T+\mathcal{D}$. If $T$ has nonvoid interior, then there exists a subset $\mathcal{J} \subseteq \mathbb{Z}^{n}$ such that

$$
T+\mathcal{J}=\mathbb{R}^{n} \text { and } \quad(T+t)^{o} \cap\left(T+t^{\prime}\right)^{o}=\emptyset, \quad t \neq t^{\prime}, t, t^{\prime} \in \mathcal{J}
$$

(see, e.g., [LW1). $T$ is called an integral self-affine tile and $\mathcal{J}$ a tiling set; $T+\mathcal{J}$ is called a tiling of $\mathbb{R}^{n}$ and a lattice tiling if $\mathcal{J}$ is a lattice.

Recently the class of self-affine tiles has attracted a lot of attention due to its special roles in fractal geometry, wavelet theory and the spectral set problem of Fuglede. Despite the extensive studies, there are still many aspects of the self-affine tiles that are not fully understood. In particular, there is very limited knowledge on the basic topological properties such as the connectedness or the disklikeness (homeomorphic to the closed unit disc), and these are the main considerations in this paper.

In $\mathrm{O}$, Odlyzko gave a characterization of a tile $T$ in $\mathbb{R}$ to be a finite union of intervals by using the strict product form digit sets. For the connectedness of the self-affine tiles $T$ in $\mathbb{R}^{n}$, the only complete result is that all 2-digit tiles are pathwise

Received by the editors October 14, 2004 and, in revised form, June 23, 2005.

2000 Mathematics Subject Classification. Primary 52C20, 52C22; Secondary 28A80.

Key words and phrases. Digit sets, neighbors, nodal points, radix expansion, self-affine tiles.

This research was partially supported by an HK RGC grant.

(C)2007 American Mathematical Society Reverts to public domain 28 years from publication 
connected (actually such tiles can be filled up by space filling curves)( $\mathrm{HSV}$, [B], Ba]). In AT1, AT2, AT3, Gi1, and Gi2, this connectedness and related properties for $T$ in $\mathbb{R}^{2}$ were studied in the context of canonical number systems. Furthermore in $[\mathrm{H}],[\mathrm{LAT}],[\mathrm{LRT}]$ and $[\mathrm{NT}]$, a more general setting for fractal sets was considered.

In view of the simplicity of the connected self-affine tiles in $\mathbb{R}^{1}$ (intervals) that are generated by $A=[q]$ and $\mathcal{D}=\{0,1,2, \cdots,|q|-1\}$, Kirat and Lau $\mathrm{KL}$ studied the connectedness of the special class of integral self-affine tiles $T$ that are generated by the consecutive collinear digit sets of the form $\{0, v, 2 v, \cdots,(|q|-1) v\}, v \in \mathbb{Z}^{n} \backslash\{0\}$. They introduced an algebraic criterion, called the height reducing property, on the characteristic polynomial $f(x)$ of $A$ to determine the connectedness of $T(A, \mathcal{D})$. In KLR, this property was further studied and it was proved that such $T$ in $\mathbb{R}^{4}$ are connected. By using this criterion, Akiyama and Gjini [AG] proved that

Theorem 1.1 ([AG]). If $T$ is a self-affine tile in $\mathbb{R}^{n}, n \leq 4$, generated by an expanding integer matrix $A$ with $\operatorname{det} A=q$ and collinear digit set $\mathcal{D}=\{0, v, 2 v, \cdots$, $(|q|-1) v\}, v \in \mathbb{Z}^{n} \backslash\{0\}$, then $T$ is connected.

The question of disklikeness was first investigated by Bandt and Gelbrich BG] for self-affine tiles in $\mathbb{R}^{2}$ with $|\operatorname{det} A|=2$ or 3 . It is clear that hexagons and squares are disklike. Recently Bandt and Wang [BW] proved, in terms of the neighbors of $T$, that the converse also holds. A translation of the tile $T+\ell, \ell \in \mathcal{J}$ is said to be a neighbor of $T$ if $T \cap(T+\ell) \neq \emptyset$.

Theorem 1.2 ([BG], $[\mathrm{BW}])$. Let $T$ be a disklike tile and $T+\mathcal{J}$ a lattice tiling of $\mathbb{R}^{2}$. Then one of the following holds.

(i) $T$ has exactly 6 neighbors $T \pm \ell_{1}, T \pm \ell_{2}, T \pm\left(\ell_{1}+\ell_{2}\right)$ for some $\ell_{1}, \ell_{2} \in \mathcal{J}$ and $\ell_{1} \mathbb{Z}+\ell_{2} \mathbb{Z}=\mathcal{J}$.

(ii) $T$ has exactly 8 neighbors $T \pm \ell_{1}, T \pm \ell_{2}, T \pm\left(\ell_{1}+\ell_{2}\right), T \pm\left(\ell_{1}-\ell_{2}\right)$ for some $\ell_{1}, \ell_{2} \in \mathcal{J}$, and $\ell_{1} \mathbb{Z}+\ell_{2} \mathbb{Z}=\mathcal{J}$.

Theorem 1.3 ([BW] $)$. Let $T$ be a tile with not more than six neighbors. Then $T$ is disklike if and only if $T$ is connected.

Note that the tiles of the first and the second cases in Theorem 1.2 are topological deformations of the hexagonal and square tiles respectively. We say that $T$ is homeomorphic to a hexagonal tile in case (i) and homeomorphic to a square tile in case (ii). The characterizations are sharp; however, there is no general criterion to determine the number of neighbors of $T$ in terms of $A$ and $\mathcal{D}$.

The main purpose of this paper is to study the disklikeness of the special class of self-affine tiles in $\mathbb{R}^{2}$ that are generated by the consecutive collinear digit sets as in $\left[\mathrm{KL}\right.$. It is known by $\left[\mathrm{BG}\right.$ that for an expanding matrix $A \in M_{2}(\mathbb{Z})$, the characteristic polynomial of $A$ is given by

$$
f(x)=x^{2}+p x+q, \quad \text { with } \quad|p| \leq q \quad \text { if } q \geq 2 ; \quad|p| \leq|q+2| \text { if } q \leq-2 .
$$

By using Theorems 1.2, 1.3, we prove our main theorem:

Theorem 1.4. Let $A \in M_{2}(\mathbb{Z})$ be an expanding matrix with characteristic polynomial $f(x)=x^{2}+p x+q$. Then for any $\mathcal{D}=\{0, v, 2 v, \ldots,(|q|-1) v\}$ in $\mathbb{Z}^{2}$ such that $v, A v$ are independent, $T$ is a disklike tile if and only if $2|p| \leq|q+2|$.

We remark that in AT1, AT3, Akiyama and Thuswaldner gave a proof of a more restrictive result that for a tiling generated by a canonical number system 
(which requires $f(x)$ to be irreducible, $-1 \leq p \leq q$ and $q \geq 2$ ), $T$ is disklike if and only if $2 p \leq q+2$. They used two different approaches: geometry of numbers and finite automata theory. The proof here is different from theirs.

As a corollary we can also classify the hexagonal and square tiles of the above tiles.

Corollary 1.5. For the above disklike tiles, if it comes from $f(x)=x^{2}+q$, then it is homeomorphic to a square tile; otherwise it is homeomorphic to a hexagonal tile.

Our proof depends on the concept of nodal point (see Gi1, [SK]). The cardinality of nodal points and general multiple points has been studied in detail in [AT3]. Let $T+\mathcal{J}$ be a tiling. We say that $x \in \mathbb{R}^{2}$ is a nodal point if $x$ is in the intersection of $T$ and two other distinct neighbors $T+\ell_{1}, T+\ell_{2}, \ell_{1}, \ell_{2} \in \mathcal{J}$. It is seen that in the hexagonal case, $T$ has six nodal points, while in the square case it has four. When $p=0$, we prove directly that $T$ is a parallelogram and is therefore obviously disklike.

For $p \neq 0$, we divide our proof into three parts:

(i) We show that all the self-affine tiles $T$ generated by the consecutive collinear digit set have at least 6 nodal points (Theorem 3.7). The construction of such nodal points depends on the recursive radix expansions and the Hamilton-Cayley theorem $f(A)=0$ (Section 3).

(ii) For $2|p|>|q+2|$, we use the same construction to yield more nodal points (Theorem 3.7). Hence by Theorem 1.2, $T$ cannot be homeomorphic to a hexagonal or a square tile and is therefore non-disklike.

(iii) We use the lattice tiling property to describe the neighbors of $T$ and show that for $2|p| \leq|q+2|, T$ has exactly six neighbors (Propositions 4.5, 4.8). By Theorem 1.1 and Theorem $1.3 \mathrm{~T}$ is disklike.

In Figure 1, we illustrate the regions of $(p, q)$ for $T$ to be connected (Theorems 1.1 and (1.2)) and disklike (Theorem 1.4). The integral points in the shaded regions are the solutions to the inequalities in (1.2); the solutions to $2|p| \leq|q+2|$ are represented by the dots in the figure.

The techniques and results developed here have been used to study the boundaries of this class of tiles by one of the authors in his thesis $\mathrm{L}$.

\section{Preliminaries}

In this section, we prove some elementary results on the self-affine tiles generated by the consecutive collinear digit sets.

Proposition 2.1. Let $A \in M_{n}(\mathbb{Z})$ be expanding, with $q=(-1)^{n} \operatorname{det} A$ and characteristic polynomial $f(x)=x^{n}+a_{n-1} x^{n-1}+a_{n-2} x^{n-2}+\ldots+a_{1} x+q$. Let $v \in \mathbb{Z}^{n} \backslash\{0\}$ and let $\mathcal{D}=\{0, v, \cdots,(|q|-1) v\} \subset \mathbb{Z}^{n}$. Then the set $\left\{v, A v, \ldots, A^{n-1} v\right\}$ is linearly independent if and only if $T=T(A, \mathcal{D})$ is a self-affine tile.

Proof. Let $T$ be a self-affine tile. Suppose on the contrary that $\left\{v, A v, \ldots, A^{n-1} v\right\}$ is linearly dependent. By $f(A) v=0$, we can express, for $i \in \mathbb{N}, A^{-i} v=\sum_{j=0}^{m} c_{j} A^{j} v$ with $m<n-1$ and $c_{j} \in \mathbb{Q}$. Then by (1.1), $T$ is contained in a set of dimension less than $n$. Hence the Lebesgue measure of $T$ is zero and this contradicts that $T$ is a tile.

To prove the converse, we let $\mathcal{J}$ be the lattice generated by $\left\{v, A v, \ldots, A^{n-1} v\right\}$. We need to show that $\mathcal{D}$ is a complete residue system $(\bmod A)$, i.e., a complete set 


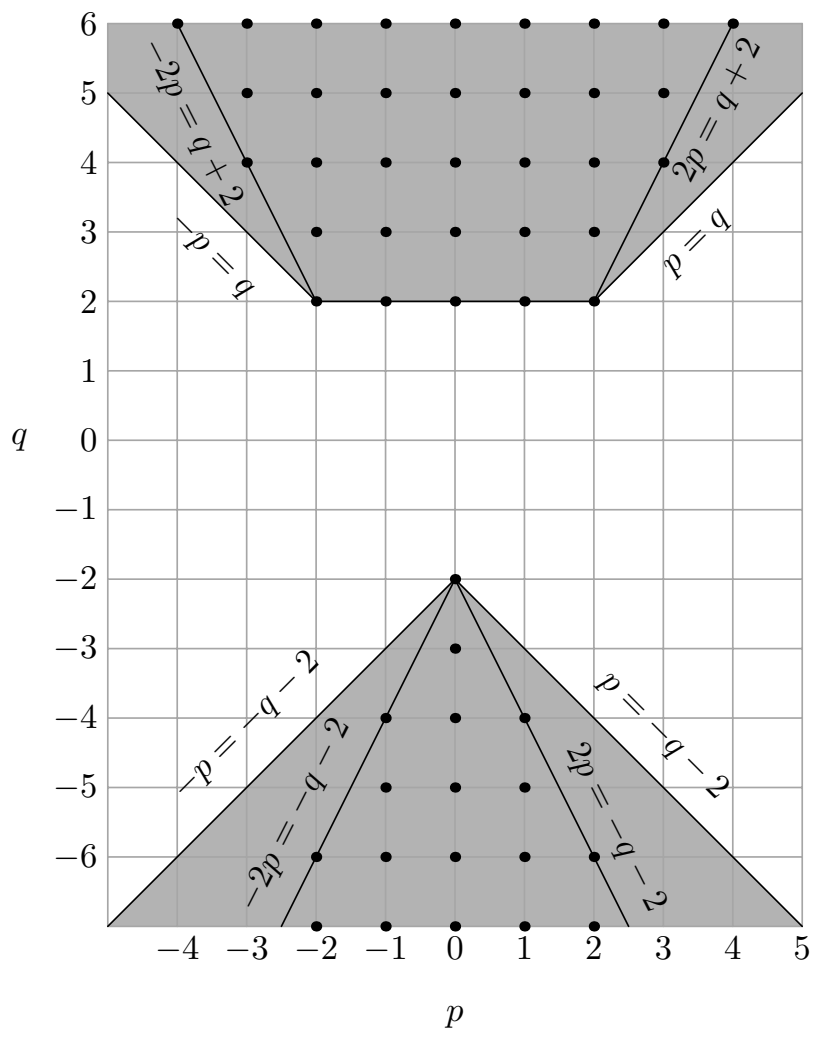

FiguRE 1. Graph of the regions of $(p, q)$ for expanding polynomials and disklike tiles

of coset representatives of the additive group $\mathcal{J} / A \mathcal{J}$; then $T$ is a tile ([B], $\mathrm{KL}]$ ). Since $f(A) v=0$, we have

$$
q v=-\sum_{i=1}^{n-1} a_{i} A^{i} v-A^{n} v \in A \mathcal{J} .
$$

For any $u \in \mathcal{J}$, write $u=\sum_{i=0}^{n-1} b_{i} A^{i} v$. Let $b_{0}=k q+r$ where $0 \leq r \leq|q|-1$. Then

$$
u=(k q+r) v+\sum_{i=1}^{n-1} b_{i} A^{i} v \in r v+A \mathcal{J}
$$

and $[u]=[r v] \in \mathcal{J} / A \mathcal{J}$. We claim that all the $[r v], 0 \leq r \leq|q|-1$, are distinct. Indeed for $w \in\left(A \mathcal{J}+r_{1} v\right) \cap\left(A \mathcal{J}+r_{2} v\right), 0 \leq r_{1}, r_{2} \leq|q|-1$, we can write

$$
w=r_{1} v+\sum_{i=1}^{n-1} b_{i} A^{i} v=r_{2} v+\sum_{i=1}^{n-1} b_{i}^{\prime} A^{i} v .
$$

By subtraction, $g(A) v=0$ where $g(x)=\left(r_{1}-r_{2}\right)+\sum_{i=1}^{n-1}\left(b_{i}-b_{i}^{\prime}\right) x^{i}$. This implies that $g(A)\left(A^{i} v\right)=0,0 \leq i \leq n-1$. The independence of $\left\{v, A v, \ldots, A^{n-1} v\right\}$ implies that $g(A)=0$. The independence also implies that the minimal polynomial of $A$ is identical to its characteristic polynomial. Hence $f(x) \mid g(x)$. This implies that 
$q \mid\left(r_{1}-r_{2}\right)$, so that $r_{1}=r_{2}$. Therefore $\mathcal{D}=\{0, v, \ldots,|q|-1\}$ is a complete coset representation of $\mathcal{J} / A \mathcal{J}$.

We remark that we can assume without loss of generality that the digit set $\mathcal{D}$ is primitive, i.e., the lattice $\mathcal{J}$ is generated by $\left\{A^{i} \mathcal{D}\right\}_{i=0}^{n-1}$ in $\mathbb{Z}^{n}$. For otherwise, there exists an invertible $B \in M_{n}(\mathbb{Z})$ such that $\tilde{\mathcal{D}}=B^{-1} \mathcal{D} \subset \mathbb{Z}^{n}$ is primitive and $T(A, D)=B T(\tilde{A}, \tilde{\mathcal{D}})$ where $\tilde{A}=B^{-1} A B \in M_{n}(\mathbb{Z})[$ LW2 and we can consider $\tilde{A}, \tilde{\mathcal{D}}$ instead.

In the following we need the concept of a stretched tile introduced by Lagarias and Wang [W] : $T$ is generated by $A$ and $\mathcal{D}$ satisfying

$A=\left[\begin{array}{cc}A_{1} & 0 \\ B & A_{2}\end{array}\right], \quad \mathcal{D}=\left\{\left[\begin{array}{c}a_{i} \\ b_{i}\end{array}\right]+\left[\begin{array}{c}0 \\ Q c_{i j}\end{array}\right]: 1 \leq i \leq\left|\operatorname{det} A_{1}\right|, 1 \leq j \leq\left|\operatorname{det} A_{2}\right|\right\}$

where $A_{1} \in M_{r}(\mathbb{Z}), A_{2} \in M_{s}(\mathbb{Z})$ such that (i) $\left\{a_{i}\right\}_{i} \subseteq \mathbb{Z}^{r}$ is a complete residue system $\left(\bmod A_{1}\right)$; (ii) $\left\{b_{i}\right\}_{i} \subset \mathbb{Z}^{s}$; (iii) $|\operatorname{det} Q| \geq 2$ and for each $i,\left\{Q c_{i, j}\right\} \subset \mathbb{Z}^{s}$ is a complete residue system $\left(\bmod A_{2}\right)$. A primitive digit set $\mathcal{D}$ is said to be standard if it is a complete residue system $(\bmod A)$. We say that two tiles $T=T(A, \mathcal{D})$ and $T^{\prime}=T\left(A^{\prime}, \mathcal{D}^{\prime}\right)$ are $\mathbb{Z}$-similar if there exists a unimodular matrix $P \in M_{n}(\mathbb{Z})$ (i.e. $P$ is invertible and $|\operatorname{det}(P)|=1$ ) such that $A^{\prime}=P A P^{-1}$ and $\mathcal{D}^{\prime}=P \mathcal{D}$. In [LW3], it was proved that if $T$ is a tile generated by a standard digit set, then $\mu(T)>1$ ( $\mu$ is the Lebesgue measure) if and only if $T$ is $\mathbb{Z}$-similar to a stretched tile.

Proposition 2.2. Assume in addition that $\mathcal{D}=\{0, v, \cdots,(|q|-1) v\} \subset \mathbb{Z}^{n}$ is primitive. Then $\mu(T)=1$ and $T+\mathbb{Z}^{n}$ is a tiling of $\mathbb{R}^{n}$.

Proof. It is known that if $\mu(T)=1$, then $\mathbb{Z}^{n}$ is a tiling set of $T$ ([LW1, [LW2]) and the second part follows. To show that $\mu(T)=1$, we assume to the contrary that $\mu(T)>1$. Since $\mathcal{D}$ is primitive and is a complete residue system $(\bmod A)$ (by Proposition 2.1), $T$ is $\mathbb{Z}$-similar to a stretched self-affine tile LW3. Without loss of generality, we assume that $A$ and $\mathcal{D}$ are the pair in the stretched tiles. Let $0 \leq i \leq\left|\operatorname{det} A_{1}\right|$. Then

$$
\left[\begin{array}{c}
a_{i} \\
b_{i}
\end{array}\right]+\left[\begin{array}{c}
0 \\
Q c_{i j}
\end{array}\right]=t_{i, j} v:=t_{i, j}\left[\begin{array}{l}
v_{1} \\
v_{2}
\end{array}\right], \quad 0 \leq t_{i, j} \leq|q|-1 .
$$

By (iii) in the definition of a stretched tile, there exist at least two distinct $t_{i, j}$. It follows that $a_{i}=v_{1}=0$. Since this is true for all $i$, it contradicts condition (i) on $\left\{a_{i}\right\}_{i}$. We conclude that $\mu(T)=1$.

We consider the simple case of disklikeness of $T \in \mathbb{R}^{2}$ corresponding to $f(x)=$ $x^{2}+q, q \in \mathbb{Z}$. It is a parallelogram, and the proof actually holds for $n \geq 2$.

Theorem 2.3. Let $A \in M_{n}(\mathbb{Z})$ be expanding with characteristic polynomial $f(x)=$ $x^{n}+q$. Let $v \in \mathbb{Z}^{n} \backslash\{0\}$ such that $\left\{v, A v, \ldots, A^{n-1} v\right\}$ is a linearly independent set and let $\mathcal{D}=\{0, v, 2 v, \ldots,(|q|-1) v\}$. Then $T(A, \mathcal{D})$ is a parallelepiped.

Proof. We first consider the case $f(x)=x^{n}-q, q \geq 2(q \geq 2$ necessarily by the expanding property of $A$ ). From $f(A)=0$, we have $A^{-n}=q^{-1} I$. Let $y=$ $\sum_{i=1}^{\infty} a_{i} A^{-i} v \in T$, where $0 \leq a_{i} \leq q-1$ for all $i=1,2, \ldots$. Then

$$
y=\sum_{j=1}^{\infty} \sum_{k=0}^{n-1} a_{j n-k} A^{-(j n-k)}=\sum_{k=0}^{n-1}\left(\sum_{j=1}^{\infty} a_{j n-k} q^{-j}\right) A^{k} v .
$$


Note that the series $\sum_{j=1}^{\infty} a_{j n-k} q^{-j}$ is the $q$-adic expansion of some $x \in[0,1]$, and conversely, any $x \in[0,1]$ can be represented in such a form. It follows that $T=\left\{\sum_{i=0}^{n-1} s_{i} A^{i} v: 0 \leq s_{i} \leq 1\right\}$ is a parallelepiped.

For the case $f(x)=x^{n}+q, q \geq 2$, we have $A^{-n}=-q^{-1} I$. By applying the same argument, we see that

$$
y=\sum_{k=0}^{n-1}\left(\sum_{j=1}^{\infty} a_{j n-k}(-q)^{-j}\right) A^{k} v .
$$

It follows that $T=\left\{\sum_{i=0}^{n-1} s_{i} A^{i} v:-q /(q+1) \leq s_{i} \leq 1 /(q+1)\right\}$ is again a parallelepiped.

\section{NODAL POINTS AND RADIX EXPANSIONS}

In the rest of this paper we always make the following assumption unless otherwise specified. $A \in M_{2}(\mathbb{Z})$ is an integral expanding matrix whose characteristic polynomial is $f(x)=x^{2}+p x+q, p, q \in \mathbb{Z}$; we will call the polynomial expanding (the roots have moduli $>1$ ). We consider $v \in \mathbb{Z}^{2} \backslash\{0\}$ such that $\{v, A v\}$ is independent, and let $\mathcal{D}=\{0, v, \ldots,(|q|-1) v\}$. It follows from Theorem 2.1 that $T$ is a tile. Without loss of generality we also assume that $\mathcal{D}$ is primitive, i.e., the lattice generated by $\{v, A v\}$ is $\mathbb{Z}^{2}$.

For a matrix series $\sum_{i=-N}^{\infty} b_{i} A^{-i}$, we will represent it in the form of a radix expansion as

$$
b_{-N} b_{-N+1} \ldots b_{-1} b_{0} . b_{1} b_{2} b_{3} \ldots
$$

We call $b_{-N} b_{-N+1} \ldots b_{-1} b_{0}$ the integral part and $0 . b_{1} b_{2} b_{3} \ldots$ the fractional part. An overbar represents a group of repeating digits:

$$
0 . b_{1} \cdots \overline{b_{2} b_{3} b_{4}}=0 . b_{1} \cdots b_{2} b_{3} b_{4} b_{2} b_{3} b_{4} b_{2} b_{3} b_{4} \cdots
$$

When there is no confusion, we also use the same radix expansion notation to denote a vector $x=\sum_{i=-N}^{\infty} b_{i} A^{-i} v \in \mathbb{R}^{2}$. Indeed since $T+\mathbb{Z}^{2}$ is a tiling of $\mathbb{R}^{2}$, each $x \in \mathbb{R}^{2}$ can be expressed as

$$
x=b_{-1} A v+b_{0} v+\sum_{i=0}^{\infty} b_{i} A^{-i} v, \quad b_{-1}, b_{0} \in \mathbb{Z}, 0 \leq b_{i} \leq|q|-1, \quad i \geq 1 .
$$

Hence $x$ can be represented as

$$
x \stackrel{v}{=} b_{-1} b_{0} . b_{1} b_{2} b_{3} \ldots, \quad b_{-1}, b_{0} \in \mathbb{Z}, \quad 0 \leq b_{i} \leq|q|-1, i \geq 1
$$

(we omit the $v$ throughout). The algebraic operation of the radix expansion is simple: shifting a radix place to the left means multiplying $A$ to $x$; for vector addition, we can add and subtract the radix expansions. Note that the radix representation of $x$ may not be unique. This is the case when $x$ is on the boundary of $T$. In fact we have

Lemma 3.1. Suppose $x$ has two distinct representations

$$
x=0 . a_{1} a_{2} \cdots=b_{-1} b_{0} . b_{1} b_{2} \cdots .
$$

Then $x \in T \cap(T+\ell)$ with $\ell=b_{-1} A v+b_{0} v$, and $a_{i} \neq b_{i}$ for infinitely many $i$. 
Proof. The first statement is clear. To prove the second part, we observe that $a_{k} \neq b_{k}$ for at least one $k>0$. We claim that there exists $i>k$ such that $a_{i} \neq b_{i}$. Otherwise, by subtracting the two radix expansions and shifting $k$ places to the right, we have

$$
g(A) v=b_{-1} A^{k+2} v+b_{0} A^{k+1} v+\left(b_{k}-a_{k}\right) v=0 .
$$

By the same argument as in Proposition 2.1, we have that $f(x)$ divides $g(x)$. This is impossible since $\left|b_{k}-a_{k}\right|<q$ and the claim is proved. The lemma follows by repeatedly applying this argument.

Definition 3.2. A point $x \in T$ is called a nodal point if $x \in\left(T+\ell_{1}\right) \cap\left(T+\ell_{2}\right)$ for some distinct $\ell_{1}, \ell_{2} \in \mathbb{Z}^{2} \backslash\{0\}$, i.e., $x$ is in the intersection of at least three translated copies of $T$ (one copy being $T$ itself). We also say that $x$ is a $k$-nodal point if it is the intersection of $k \geq 3$ translates of $T$.

A (topological) square tile has exactly four nodal points, all of which are 4nodal. A (topological) hexagonal tile has exactly six nodal points, all of which are 3-nodal. Algebraically, $x \in \mathbb{R}^{2}$ is a $k$-nodal point if and only if it has $k$ different series expansions, no two of which have equal integral parts. We will use the following simple device to construct new nodal points from a given one.

Lemma 3.3. Suppose $0 . a_{1} a_{2} \ldots$ represents a nodal point $x$. Then $0 . a_{k} a_{k+1} \cdots, k \geq$ 1 also represents a nodal point $x_{k}$. Furthermore if $0 . a_{1} a_{2} \ldots=b_{-1} b_{0} \cdot b_{1} b_{2}$, then $0 . b_{k} b_{k+1} \ldots, k \geq 1$ also represents nodal points of $T$.

Proof. By definition $x$ has at least three representations:

$$
0 . a_{1} a_{2} \ldots=b_{-1} b_{0} . b_{1} b_{2}=c_{-1} c_{0} . c_{1} c_{2} \ldots
$$

Hence

$$
0 . a_{2} a_{3} \ldots=b_{-1} b_{0}\left(b_{1}-a_{1}\right) \cdot b_{2} b_{3}=c_{-1} c_{0}\left(c_{1}-a_{1}\right) \cdot c_{2} \ldots
$$

Note that $b_{-1} b_{0}\left(b_{1}-a_{1}\right)$ represents $b_{1} A^{2}+b_{0} A+\left(b_{1}-a_{1}\right) I$. We can use $A^{2}+p A+q=$ 0 to reduce it to $b_{-1}^{\prime} A+b_{0}^{\prime} I$. This implies that

$$
0 . a_{2} a_{3} \ldots=b_{-1}^{\prime} b_{0}^{\prime} \cdot b_{2} b_{3}=c_{-1}^{\prime} c_{0}^{\prime} \cdot c_{2} c_{3} \ldots,
$$

and they are distinct representations (Lemma 3.1). Hence $0 . a_{2} a_{3} \ldots$ represents a nodal point of $T$, and we can continue this inductively.

For the second expression, we have

$$
0 . b_{1} b_{2} \ldots=\left(-b_{1}\right)\left(-b_{0}\right) \cdot a_{1} a_{2} \cdots=\left(c_{-1}-b_{-1}\right)\left(c_{0}-b_{0}\right) \cdot c_{1} c_{2} \ldots
$$

These are distinct representations by Lemma 3.1 and hence it is also a nodal point.

The nodal points so obtained by the above method are not necessarily distinct. Nevertheless, we have

Corollary 3.4. If $x$ is a nodal point of $T$, then there are at least two more distinct nodal points arising from the construction in Lemma 3.3 .

Proof. For the representation of $x$ in (3.1), we have two more nodal points: $0 . b_{1} b_{2} \ldots$ (3.2) and $0 . c_{1} c_{2} \cdots$. They are distinct by Lemma 3.1 . 
As an illustration we consider the case that the characteristic polynomial of $A$ is $f(x)=x^{2}-q, q \geq 2, T$ is a parallelogram (Theorem 2.3) and therefore $T$ has four 4-nodal points: $0, v, A v$, and $v+A v$. To see that $v$ is a 4-nodal point in the radix expansion, we first observe that $v$ has representation 1. By $f(A)=0$, we have

$$
I=(q-1)\left(A^{2}-I\right)^{-1}=r \sum_{i=1}^{\infty} A^{-2 i}
$$

where $r=(q-1)$. This implies that $1=0 . \overline{0 r}$. If we shift the expression one radix place to the right and add $(-1) 1$, we have $1=(-1) 1 \cdot \overline{r 0}$. By adding $(-1) 0$ to $1=0 . \overline{0 r}$, we obtain

$$
1=0 . \overline{0 r}=(-1) 1 \cdot \overline{r 0}=(-1) 0 . \bar{r} .
$$

By repeating the elementary operations, we come up with the other nodal points:

$$
\begin{aligned}
0 & =(-1) \cdot \overline{0 r}=(-1) 0 \cdot \overline{r 0}=(-1)(-1) \cdot \bar{r} ; \\
11 & =10 \cdot \overline{0 r}=1 \cdot \overline{r 0}=0 . \bar{r} ; \\
10 & =1(-1) \cdot \overline{0 r}=0 \cdot \overline{r 0}=(-1) \cdot \bar{r} .
\end{aligned}
$$

For $f(x)=x^{2}+q, q \geq 2$, the four nodal points are

$$
\alpha v+\alpha A v, \quad \beta v+\alpha A v, \quad \alpha v+\beta A v, \quad \beta v+\beta A v
$$

where $\alpha=-q /(q+1)$ and $\beta=1 /(q+1)$. We can also go through a similar argument as the above: from $f(A)=0$, we obtain

$$
I=-(q-1)\left(I+A^{-2}\right) A^{-2}=r \sum_{i=1}^{\infty}(1)^{i} A^{-2 i},
$$

which yields $1=0 . \overline{0(-r) 0 r}$. By using elementary operations on the radix expansion and making use of $10 r=-1$ (i.e., $A^{2}+(q-1) I=-I$ ), we obtain

$$
0 . \overline{r 00 r}=(-1) 0 . \overline{00 r r}=1 \cdot \overline{r r 00}=(-1) 1 \cdot \overline{0 r r 0} \text {. }
$$

The other three nodal points follow from Lemma 3.3 and $10 r=-1$ :

$$
\begin{aligned}
& 0 . \overline{00 r r}=1 \cdot \overline{0 r r 0}=10 \cdot \overline{r 00 r}=11 \cdot \overline{r r 00}, \\
& 0 . \overline{0 r r 0}=10 \cdot \overline{r r 00}=(-1) \cdot \overline{00 r r}=1(-1) \cdot \overline{r 00 r}, \\
& 0 . \overline{r r 00}=(-1) \cdot \overline{r 00 r}=(-1) 0 \cdot \overline{0 r r 0}=(-1)(-1) \cdot \overline{00 r r} .
\end{aligned}
$$

Theorem 3.5. Let $A \in M_{2}(\mathbb{Z})$ be expanding and have characteristic polynomial $f(x)=x^{2}+p x+q$ and $p \neq 0$. Then the tile $T(A, \mathcal{D})$ generated by $\mathcal{D}=\{0, v, \cdots$, $(|q|-1) v\}$ has at least six nodal points.

Proof. It is more convenient to consider $f(x)=x^{2} \pm p x \pm q$ where $p, q>0$ and prove each case separately.

Case (i). $\quad f(x)=x^{2}+p x+q$ : The expanding property implies that $0<p \leq q$ (see (1.2) ). By using $0=f(A)=f(A)(A-I)=A^{3}+(p-1) A^{2}+(q-p) A-q I$, we have

$$
\begin{aligned}
I & =\left(A^{3}-I\right)^{-1}\left(-(p-1) A^{2}-(q-p) A+(q-1) I\right) \\
& =\left(\sum_{i=1}^{\infty} A^{-3 i}\right)\left(-(p-1) A^{2}-(q-p) A+(q-1) I\right) .
\end{aligned}
$$


Let $r=(p-1), s=(q-1)$. Then

$$
1=0 . \overline{(-r)(r-s) s} \text {. }
$$

We add $0 . \overline{r s 0}$; then $1 . \overline{r s 0}=0 . \overline{0 r s}$. We then apply the two operations to the identity separately: shifting one radix place to the right; subtracting 1 . Then

$$
1 r . \overline{s 0 r}=0 . \overline{r s 0}=(-1) \cdot \overline{0 r s} .
$$

Hence $0 . \overline{r s 0}$ is a nodal point, and $0 . \overline{s 0 r}$ and $0 . \overline{0 r s}$ are also nodal points by Corollary 3.4.

We need to find three more nodal points. Let $t=s-r$ and add $0 . \overline{s t 0}$ to (3.3); then $1 . \overline{s t 0}=0 . \overline{t 0 s}$. By using the same technique as the above, we can find another representation $(-1)(-r) . \overline{0 s t}$. Hence $0 . \overline{t 0 s}$ is a nodal point; there are two more following from Corollary 3.4. Note that these three are distinct from the previous three as $0 . \overline{r s 0} \neq 0 . \overline{s t 0}$.

Case (ii). $f(x)=x^{2}-p x+q$ : Again $0<p \leq q$ by (1.2). By using $0=f(A)=$ $f(A)(A+I)=A^{3}-(p-1) A^{2}+(q-p) A+q I$, we have

$$
\begin{aligned}
I & =\left(A^{3}+I\right)^{-1}\left((p-1) A^{2}-(q-p) A-(q-1) I\right) \\
& =\left(\sum_{i=1}^{\infty}(-A)^{-3 i}\right)\left((p-1) A^{2}-(q-p) A+(q-1) I\right) .
\end{aligned}
$$

Let $r=(p-1), s=(q-1), t=(r-s)$. Then the sequence representation for 1 is

$$
1=0 . \overline{r(-t)(-s)(-r) t s} .
$$

Adding $0 . \overline{0 t s s r} 0$ yields $1 . \overline{0 t s s r} 0=0 . \overline{r 00 t s s}$. Following by shifting two places to the right, adding $1 q(q-1)$ and making use of $1(-p) q=0$, we have

$$
0 . \overline{r 00 t s s}=1 . \overline{0 t s s r 0}=1(-t) \cdot \overline{t s s r 00} \text {. }
$$

By using Lemma 3.3, we can produce six nodal points by cyclic permutations.

Case (iii). $f(x)=x^{2}+p x-q$ : The expanding property implies that $p \leq q-2$ (by (1.2)). From $f(A)=0$ and $I=\left(A^{2}-I\right)^{-1}(-p A+(q-1) I)$, we have

$$
1=0 . \overline{(-p)(q-1)} \text {. }
$$

By adding $0 . \overline{p 0}$ and $0 . \overline{(p-1) 0}$ to the identity separately, we have

$$
1 . \overline{p 0}=0 . \overline{0(q-1)} \text { and } 1 . \overline{(q-1) 0}=0 . \overline{(q-1-p)(q-1)} .
$$

By shifting the last expression one place to the right and by subtracting $1(q-1)$, we have

$$
1 \cdot \overline{p 0}=0 . \overline{0(q-1)}=(-1)(-p) \cdot \overline{(q-1)(q-1-p)} .
$$

By Lemma 3.3 again, we get six nodal points.

Case (iv). $f(x)=x^{2}-p x-q$ : The expanding property implies $p \leq q-2$. We adopt a similar proof as in case (iii). From $f(A)=0$, we have

$$
1=0 . \overline{p(q-1)} \text {. }
$$

By shifting one place to the right and adding $(-1) 1$, we obtain

$$
1 \cdot \overline{0}=(-1)(p+1) \cdot \overline{(q-1) p}=0 \cdot \overline{p(q-1)} .
$$

By Corollary 3.4, we get two more nodal points. To obtain three more, we add $0 . \overline{(q-1-p) 0}$ to (3.4); then

$$
1 . \overline{(q-1-p)}=0 \cdot \overline{(q-1)} .
$$


By shifting one place to the right and subtracting $(q-1)$, we have

$$
0 . \overline{(q-1)}=1(-p) \cdot \overline{0(q-1-p)} \text {. }
$$

Hence $0 . \overline{(q-1)}$ is the fourth nodal point, and we get two more by Corollary 3.4 .

Corollary 3.6. Under the assumptions of the above theorem, $T$ has at least six neighbors.

Proof. By writing down explicitly all the radix expansions for all the nodal points obtained in cases (i) to (iv) in the above theorem, we can find six different non-zero integral parts in each case. Each non-zero integral part $l$ corresponds to a neighbor $T+l$ of $T$.

Case (i). $f(x)=x^{2}+p x+q$ : The six non-zero integral parts obtained in Theorem 3.5 are

$$
1,1(p-1), 1 p, 1 p(q-1), 1(p-1)(q-p+1), 1(p-1)(q-p) .
$$

We can express them in the form $\alpha v+\beta A v$ by using $f(A) v=0$ :

$$
\pm v, \pm[A v+(p-1) v], \pm(A v+p v) .
$$

Case (ii). $f(x)=x^{2}-p x+q$ : The six non-zero integral parts so obtained are

$$
\pm v, \pm[A v-(p-1) v], \pm(A v-p v) .
$$

Case (iii). $f(x)=x^{2}+p x-q$ : The six non-zero integral parts so obtained are

$$
\pm v, \pm[A v+(p+1) v], \pm(A v+p v) \text {. }
$$

Case (iv). $f(x)=x^{2}-p x-q$ : The six non-zero integral parts so obtained are

$$
\pm v, \pm[A v-(p+1) v], \pm(A v-p v) .
$$

Our main theorem in this section is

Theorem 3.7. Let $A \in M_{2}(\mathbb{Z})$ be expanding and let $f(x)=x^{2}+p x+q$ be the characteristic polynomial of $A$. Suppose $2|p|>|q+2|$. Then the tiles $T(A, \mathcal{D})$ with $\mathcal{D}=\{0, v, \cdots(|q|-1) v\}$ are non-disklike.

Proof. We need to find one more nodal point in addition to those in Theorem 3.5 then by Corollary 3.4 there are at least 9 nodal points. Theorem 1.2 implies that $T$ is non-disklike.

Case (i). $f(x)=x^{2}+p x+q$ and $2 p>q+2$. From $f(A)=0$, we have $A+(p-1) I=-(q-p+1)(A+I)^{-1}$. It follows that

$$
1(p-1)=0 . \overline{(-(q-p+1))(q-p+1)} \text {. }
$$

From $p \leq q$ (by (1.2)) and $2 p>q+2$, we see that $2 \leq(2 q-2 p+2)<q$. Adding $0 . \overline{(2 q-2 p+2) 0}$ to the above equality, we get

$$
1(p-1) \cdot \overline{(2 q-2 p+2) 0}=0 . \overline{(q-p+1)} .
$$

Shifting the radix point one place to the right and then subtracting $(q-p+1)$, we have

$$
1(p-1)(q-p+1) \cdot \overline{0(2 q-2 p+2)}=0 . \overline{(q-p+1)} .
$$

The two expressions yield a nodal point $0 . \overline{(q-p+1)}$ different from the previous six. 
Case (ii). $f(x)=x^{2}-p x+q$ and $2 p>q+2$ : From $f(A)=0$ we have $A-(p-1) I=-(q-p+1)(A-I)^{-1}$. It follows that $1(-(p-1))=0 . \overline{(-(q-p+1))}$ so that

$$
1(-(p-1)) \cdot \overline{(q-p+1)}=0 .
$$

Adding $1(-(p-1)) \cdot \overline{(q-p+1)}$ and noting that $2 A-2(p-1)=A^{2}-(p-2) A+$ $(q-2 p+2)$, we have

$$
1(-(p-2))(q-2 p+2) \cdot \overline{(2 q-2 p+2)}=1(-(p-1)) \cdot \overline{(q-p+1)}=0 .
$$

Hence 0 is the additional nodal point.

Case (iii). $f(x)=x^{2}+p x-q$ and $2 p>q-2$ : From $f(A)=0$, we have $A+(p+1) I=(q-p-1)(A-I)^{-1}$. Hence

$$
1(p+1)=0 \cdot \overline{(q-p-1)}
$$

Adding $1(p+1)$ and $1(p+1) \cdot \overline{(q-p-1)}$ and noting that $1(p+1)(-(q-p))(-q)=0$, we get the new nodal point $0 . \overline{(2 q-2 p-2)}$ :

$$
1(p+1)(-(q-p-2))(2 p-q+2)=1(p+1) \cdot \overline{(q-p-1)}=0 . \overline{(2 q-2 p-2)} .
$$

Case (iv). $f(x)=x^{2}-p x-q$ and $2 p>q-2$ : From $f(A)=0$ we obtain $A-(p+1) I=(q-p-1)(A+I)^{-1}$, i.e., $1[-(p+1)]=0 . \overline{(q-p-1)(-(q-p-1))}$. Adding $(-1)(p+1) \cdot \overline{(q-p-1)}$ we have

$$
0 . \overline{(q-p-1)}=(-1)(p+1) \cdot \overline{(2 q-2 p-2) 0} .
$$

On the other hand, if we shift the radix points in the above expression one place to the right and subtract $(q-p-1)$, then

$$
0 . \overline{(q-p-1)}=1[-(p+1)] . \overline{0(2 q-2 p-2)} .
$$

Hence $0 . \overline{(q-p-1)}$ is a new nodal point.

Remark. We can also see the non-disklikeness of the $T$ by the explicit expression of the neighbors in Theorem 1.2. For example we consider the case when $f(x)=$ $x^{2}+p x+q$ and $2 p>q+2$. By Corollary 3.6 and Theorem 3.7, we find that the $\ell$ in the neighbors $T+\ell$ is equal to

$$
\pm v, \quad \pm(A v+(p-1) v), \quad \pm(A v+p v), \quad \pm(2 A v+(2 p-2) v) .
$$

These neighbors are not arranged in the pattern in Theorem 1.2 hence $T$ is nondisklike.

\section{Proof of the main theorem}

Let $f(x)=x^{2}+p x+q$ be the characteristic polynomial of $A \in M_{2}(\mathbb{Z})$. As we have already dealt with the case $p=0$ (Theorem [2.3), in this section, we will assume $p \neq 0$ unless otherwise mentioned. Our main theorem is

Theorem 4.1. Let $A \in M_{2}(\mathbb{Z})$ be expanding and let $f(x)=x^{2}+p x+q$ be the characteristic polynomial of $A$. Suppose $0<2|p| \leq|q+2|$. Then the tiles $T(A, \mathcal{D})$ with $\mathcal{D}=\{0, v, \cdots(|q|-1) v\}$, are homeomorphic to a hexagonal tile. 
The most essential part in the proof is to show that $T$ has exactly six neighbors. Recall that $\mathbb{Z}^{2}=\{\gamma v+\delta A v: \gamma, \delta \in \mathbb{Z}\}$ and $T+\mathbb{Z}$ is a lattice tiling (Proposition 2.2). It is easy to see that $T+\ell$ is a neighbor of $T$ if and only if

$$
\ell=\gamma v+\delta A v=\left\{\sum_{i=1}^{\infty} b_{i} A^{-i} v: b_{i} \in \mathbb{Z} \text { and }\left|b_{i}\right| \leq|q|-1\right\} \in T-T ;
$$

also $T+\ell$ is a neighbor of $T$ if and only if $T-\ell$ is a neighbor of $T$.

For the characteristic polynomial $f(x)=x^{2}+p x+q$ where $p, q \neq 0$, let $\Delta=p^{2}-4 q$ be the discriminant. Define $\alpha_{i}$ and $\beta_{i}$ by

$$
A^{-i} v=\alpha_{i} v+\beta_{i} A v, \quad i=1,2, \ldots
$$

The following result plays an important role in our proof.

Lemma 4.2. Let $\alpha_{i}$ and $\beta_{i}$ be defined as above. Then for $i \geq 1$,

$$
q \alpha_{i+2}+p \alpha_{i+1}+\alpha_{i}=0, \quad q \beta_{i+2}+p \beta_{i+1}+\beta_{i}=0 .
$$

Moreover,

(i) for $\Delta \neq 0$, we have

$$
\alpha_{i}=q\left(y_{1}^{i+1}-y_{2}^{i+1}\right) / \Delta^{1 / 2}, \quad \beta_{i}=-\left(y_{1}^{i}-y_{2}^{i}\right) / \Delta^{1 / 2}
$$

where $y_{1}=\left(-p+\Delta^{1 / 2}\right) /(2 q)$ and $y_{2}=\left(-p-\Delta^{1 / 2}\right) /(2 q)$ are the two roots of $q x^{2}+p x+1$

(ii) for $\Delta=0$, we have

$$
\alpha_{i}=(i+1) y^{i}, \quad \beta_{i}=2 i y^{i} / p,
$$

where $y=-p /(2 q)$.

Proof. Putting the expression of $A^{-1} v$ and $A^{-i} v=\alpha_{i} v+\beta_{i} A v$ into $A^{-(i+1)} v=$ $\alpha_{i+1} v+\beta_{i+1} A v$, we get

$$
\alpha_{i+1} v+\beta_{i+1} A v=\alpha_{i} A^{-1} v+\beta_{i} v=-\alpha_{i} q^{-1} A v+\left(-p q^{-1}+\beta_{i}\right) v .
$$

It follows that $\alpha_{i+1}=-p q^{-1} \alpha_{i}+\beta_{i}$ and $\beta_{i+1}=-q^{-1} \alpha_{i}$. Then by direct calculation, we obtain

$$
q \alpha_{i+2}+p \alpha_{i+1}+\alpha_{i}=0, \quad q \beta_{i+2}+p \beta_{i+1}+\beta_{i}=0 .
$$

When $\Delta \neq 0$, the general solutions are

$$
\alpha_{i}=c y_{1}^{i}+d y_{2}^{i}, \quad \beta_{i}=c^{\prime} y_{1}^{i}+d^{\prime} y_{2}^{i} .
$$

It follows from $f(A) v=0$ that $v=q^{-1}\left(-A^{2} v-p A v\right)$; hence

$$
A^{-1} v=q^{-1}(-A v-p v) \quad \text { and } \quad A^{-2} v=q^{-2}\left(p^{2}-q\right) v+p q^{-2} A v .
$$

We have

$$
\alpha_{1}=-p q^{-1}, \quad \beta_{1}=-q^{-1} ; \quad \alpha_{2}=q^{-2}\left(p^{2}-q\right), \quad \beta_{2}=p q^{-2} .
$$

We can solve the constants in (4.5) and obtain the expression in (4.3).

When $\Delta=0$, the general solutions for the two recurrence relations are $\alpha_{i}=$ $c y^{i}+i d y^{i}$ and $\beta_{i}=c^{\prime} y^{i}+i d^{\prime} y^{i}$, and we can use the same method to obtain (4.4). 
Since the characteristic polynomial $f(x)=x^{2}+p x+q$ is expanding, the two roots $y_{1}, y_{2}$ of $x^{2} f\left(x^{-1}\right)=q x^{2}+p x+1$ have moduli less than 1 . This implies that the following two series converge:

$$
\widetilde{\alpha}=\sum_{i=1}^{\infty}\left|\alpha_{i}\right| \text { and } \widetilde{\beta}=\sum_{i=1}^{\infty}\left|\beta_{i}\right| .
$$

Corollary 4.3. Let $f(x)=x^{2}+p x+q$ and $g(x)=x^{2}-p x+q$ be expanding polynomials. Let $\alpha_{i}, \beta_{i}, \widetilde{\alpha}$ and $\widetilde{\beta}$ for $f(x)$ be as before; let $\alpha_{i}^{\prime}, \beta_{i}^{\prime}$, $\widetilde{\alpha}^{\prime}$ and $\widetilde{\beta}^{\prime}$ be the corresponding terms for $g(x)$. Then

$$
\alpha_{2 j}^{\prime}=\alpha_{2 j}, \quad \alpha_{2 j-1}^{\prime}=-\alpha_{2 j-1}, \quad \beta_{2 j}^{\prime}=-\beta_{2 j}, \quad \beta_{2 j-1}^{\prime}=\beta_{2 j-1},
$$

and hence, $\widetilde{\alpha}=\widetilde{\alpha}^{\prime}, \quad \widetilde{\beta}=\widetilde{\beta^{\prime}}$.

Lemma 4.4. Let $T+\ell$ be a neighbor of $T$ with $\ell=\gamma v+\delta A v, \gamma$ and $\delta$ not both zero. Then $|\gamma| \leq(|q|-1) \widetilde{\alpha}$ and $|\delta| \leq(|q|-1) \widetilde{\beta}$. Furthermore if $|q|-|p|-1>0$, then

$$
|\delta| \leq(|q|-1) /(|q|-|p|-1) .
$$

Proof. Note that by (4.1),

$$
\ell=\gamma v+\delta A v=\sum_{i=1}^{\infty} b_{i} A^{-i} v=\sum_{i=1}^{\infty} b_{i}\left(\alpha_{i} v+\beta_{i} A v\right) .
$$

The first part follows from this expression and $\left|b_{i}\right| \leq|q|-1$. For the second part we make an estimation of $\widetilde{\beta}$. By Lemma 4.2, we have $q \beta_{i+2}+p \beta_{i+1}+\beta_{i}=0$, and hence $|q|\left|\beta_{i+2}\right| \leq|p|\left|\beta_{i+1}\right|+\left|\beta_{i}\right|$. Summing the inequality for $i \geq 1$, we get $|q|\left(\widetilde{\beta}-\left|\beta_{1}\right|-\left|\beta_{2}\right|\right) \leq|p|\left(\widetilde{\beta}-\left|\beta_{1}\right|\right)+\widetilde{\beta}$. Note that $\left|\beta_{1}\right|=1 / q,\left|\beta_{2}\right|=p / q^{2}$ (by (4.6) $)$. It follows that

$$
(|q|-|p|-1) \widetilde{\beta} \leq(|q|-|p|)\left|\beta_{1}\right|+|q|\left|\beta_{2}\right|=1 .
$$

We conclude that $\widetilde{\beta} \leq 1 /(|q|-|p|-1)$.

We need the estimate that $|\delta| \leq 1$. It will be proved in two propositions for the cases $\Delta \geq 0$ and $\Delta<0$.

Proposition 4.5. Suppose $f(x)=x^{2}+p x+q$ with $0<2|p| \leq|q+2|$ and $\Delta \geq 0$. Let $T+\ell$ be a neighbor of $T$ with $\ell=\gamma v+\delta A v$; then $|\delta| \leq 1$.

Proof. We will use the estimate of $\delta$ by $\widetilde{\beta}$ in Lemma 4.4. Since the $\widetilde{\beta}$ are the same for $x^{2}-p x+q$ and $x^{2}+p x+q$, we can assume without loss of generality that $p>0$. We divide the proof into three cases.

Case (i). $f(x)=x^{2}+p x-q$ where $p, q>0$ : In this case $\Delta>0$ always; the condition on $p, q$ becomes $0<2 p \leq q-2$. It follows from the assumption that $q-p-1>0$. By Lemma 4.4, we have

$$
|\delta| \leq(q-1) /(q-p-1)<(q-1) /[q-(q-2) / 2-1]<2 .
$$

Case (ii). $f(x)=x^{2}+p x+q$ where $p, q>0$ and $\Delta=p^{2}-4 q>0$. In view of Lemma 4.2, both $y_{1}=\left(-p+\Delta^{1 / 2}\right) /(2 q)$ and $y_{2}=\left(-p-\Delta^{-1 / 2}\right) /(2 q)$ are negative and $\left|y_{1}\right|<\left|y_{2}\right|<1$. Hence we have

$$
\beta_{i}=\frac{-\left(y_{1}^{i}-y_{2}^{i}\right)}{\Delta^{1 / 2}} \begin{cases}<0, & \text { if } i \text { is odd } \\ >0, & \text { if } i \text { is even }\end{cases}
$$


Let $\beta_{E}=\sum_{i=1}^{\infty} \beta_{2 i}$ and $\beta_{O}=\sum_{i=1}^{\infty} \beta_{2 i-1}$. By summing the relation $q \beta_{i+2}+p \beta_{i+1}+$ $\beta_{i}=0$ for the even and odd $i$ 's respectively, we obtain

$$
q\left(\beta_{E}-\beta_{2}\right)+p\left(\beta_{O}-\beta_{1}\right)+\beta_{E}=0, \quad q\left(\beta_{O}-\beta_{1}\right)+p \beta_{E}+\beta_{O}=0 .
$$

Solving these two equations, we get

$$
\beta_{E}=\frac{p}{(q+1)^{2}-p^{2}}, \quad \beta_{O}=\frac{-(q+1)}{(q+1)^{2}-p^{2}} .
$$

By Lemma 4.4 and $\tilde{\beta}=\beta_{E}-\beta_{O}$, we have

$$
|\delta| \leq(q-1)\left(\beta_{E}-\beta_{O}\right)=\frac{q-1}{q-p+1} \leq \frac{q-1}{q-(q / 2+1)+1}=2-2 / q .
$$

Case (iii). $f(x)=x^{2}+p x+q$ where $q>0$ and $\Delta=p^{2}-4 p=0$. By Lemma4.2, $\tilde{\beta}=4(p-2)^{-2}$. Also we see that $p^{2}=4 q$ and $2 p \leq q+2$ imply $p \geq 8$. Hence

$$
|\delta| \leq(q-1) \tilde{\beta}=4(q-1) /(p-2)^{2}=1+4 /(p-2)<2 .
$$

The above method does not work for $\Delta=p^{2}-4 q<0$ as $\tilde{\beta} \neq \beta_{E}-\beta_{O}$. We use a different estimation for $\delta$ in this case. We need two lemmas. The first one is a special case in Lemma 3.3 of constructing new neighbors from a given one:

Lemma 4.6. Let $A \in M_{2}(\mathbb{Z})$ be an expanding matrix with characteristic polynomial $f(x)=x^{2}+p x+q$ where $p, q \in \mathbb{Z}$. Let $\ell=\gamma v+\delta A v=\sum_{i=1}^{\infty} b_{i} A^{-i} v(\neq 0)$ such that $T+\ell$ is a neighbor of $T$. Let

$$
\ell^{\prime}=\gamma^{\prime} v+\delta^{\prime} A v \quad \text { with } \quad \gamma^{\prime}=-\left(q \delta+b_{1}\right), \quad \delta^{\prime}=\gamma-p \delta .
$$

Then $\ell^{\prime} \neq 0$ and $T+\ell^{\prime}$ is also a neighbor of $T$.

Proof. We write $\ell$ in the radix expansion: $\ell=\delta \gamma=0 . b_{1} b_{2} \cdots$. By a simple algebraic operation and given that $100=(-p)(-q)$ (i.e., $\left.A^{2} v+p A v+q v=0\right)$, we have

$$
0 . b_{2} \cdots=\delta \gamma\left(-b_{1}\right)=(\gamma-p \delta)\left(-q \delta-b_{1}\right):=\delta^{\prime} \gamma^{\prime}=\ell^{\prime} .
$$

The lemma will follow if we can show that $\gamma^{\prime}$ and $\delta^{\prime}$ are not both zero. Indeed if $\delta \neq 0$, then $\left|\gamma^{\prime}\right|=\left|q \delta+b_{1}\right| \geq|q||\delta|-\left|b_{1}\right| \geq|q|-(|q|-1)=1$; if $\delta=0$, we have $\delta^{\prime}=\gamma \neq 0$.

Remark. We can use the above construction repeatedly to obtain a sequence $\left\{\ell_{k}\right\}_{k=0}^{\infty}$ with $\ell_{0}=\ell, \ell_{k}=\gamma_{k} v+\delta_{k} A v$, and

$$
\gamma_{k+1}=-\left(q \delta_{k}+b_{k+1}\right) \text { and } \delta_{k+1}=\gamma_{k}-p \delta_{k} .
$$

These $T+\ell_{k}$ are neighbors of $T$ but not all distinct.

The following is a refinement of Lemma 4.4

Lemma 4.7. Let $f(x)=x^{2}+p x+q$ be the characteristic polynomial of $A$ and $\Delta=p^{2}-4 q<0$. Then for $j>1$,

$$
\begin{gathered}
|\gamma| \leq(q-1)\left(\sum_{i=1}^{j-1}\left|\alpha_{i}\right|+\frac{2 q^{-(j-1) / 2}}{\left(1-q^{-1 / 2}\right)\left(4 q-p^{2}\right)^{1 / 2}}\right) \leq \frac{2 q^{1 / 2}\left(q^{1 / 2}+1\right)}{\left(4 q-p^{2}\right)^{1 / 2}} \\
|\delta| \leq(q-1)\left(\sum_{i=1}^{j-1}\left|\beta_{i}\right|+\frac{2 q^{-j / 2}}{\left(1-q^{-1 / 2}\right)\left(4 q-p^{2}\right)^{1 / 2}}\right) \leq \frac{2\left(q^{1 / 2}+1\right)}{\left(4 q-p^{2}\right)^{1 / 2}}
\end{gathered}
$$


Proof. Note that $q>0$ follows from $\Delta<0$. By Lemma 4.2. $\alpha_{i}=$ $q\left(y_{1}^{i+1}-y_{2}^{i+1}\right) / \Delta^{1 / 2}$ and $\beta_{i}=-\left(y_{1}^{i}-y_{2}^{i}\right) / \Delta^{1 / 2}$, we deduce that

$$
\left|\alpha_{i}\right| \leq \frac{2 q\left|y_{1}\right|^{i+1}}{\left|\Delta^{1 / 2}\right|}=\frac{2 q^{-(i-1) / 2}}{\left(4 q-p^{2}\right)^{1 / 2}}, \quad\left|\beta_{i}\right| \leq \frac{2\left|y_{1}\right|^{i}}{\left|\Delta^{1 / 2}\right|}=\frac{2 q^{-i / 2}}{\left(4 q-p^{2}\right)^{1 / 2}} .
$$

The statement follows by summing each of these two inequalities (see the first part of the proof of Lemma 4.4).

Proposition 4.8. Suppose $f(x)=x^{2}+p x+q$ with $0<2|p| \leq|q+2|$ and $\Delta<0$. Let $T+\ell$ be a neighbor of $T$ with $\ell=\gamma v+\delta A v$; then $|\delta| \leq 1$.

Proof. Note that $\Delta<0$ implies that $q>0$. Without loss of generality we can assume that $p>0$ (Corollary 4.3 and Lemma 4.4). We divide our consideration into two cases:

Case (i). $2 p \leq q-2$ : We can make use of Lemma 4.4 to conclude that $|\delta| \leq$ $(q-1) /(q-p-1)<2$.

Case (ii). $\quad q-1 \leq 2 p \leq q+2$ : Let $p=(q+j) / 2, j=-1,0,1,2$. Since $\Delta=p^{2}-4 q<0$, the $q$ 's in the respective cases are the integers in the sets

$$
\{3,5, \ldots, 17\},\{2,4, \ldots, 14\},\{3,5, \ldots, 13\},\{2,4, \ldots, 10\} .
$$

Suppose $|\delta|>1$, we use the sharper estimate of $\gamma^{\prime}=-\left(q \delta+b_{1}\right)$ in Lemma 4.7 to obtain

$$
q+1=|(2 q-(q-1))| \leq\left|\gamma^{\prime}\right| \leq \frac{2 q^{1 / 2}\left(q^{1 / 2}+1\right)}{\left(4 q-(q+j)^{2} / 4\right)^{1 / 2}} .
$$

By a direct calculation, we have

$$
(14-2 j) q^{3}+\left(15-4 j-j^{2}\right) q^{2} \leq q^{4}+32 q^{3 / 2}+\left(2 j+2 j^{2}\right) q+j^{2} .
$$

With the exceptions of the three cases

$$
j=-1, q=17(p=8) ; \quad j=1, q=13(p=7) ; \quad j=2, q=10(p=6),
$$

all the $q$ 's in the respective sets in (4.11) do not satisfy the inequality, which leads to a contradiction; hence $|\delta| \leq 1$.

We will deal with the three exceptional cases separately. For the first case $p=8, q=17$, we obtain the values of $\beta_{i}, i=1, \ldots, 4$ from (4.2) and (4.6). It follows from Lemma 4.7 that

$$
|\delta| \leq 16\left(\frac{1}{17}+\frac{8}{17^{2}}+\frac{47}{17^{3}}+\frac{240}{17^{4}}+\frac{(17)^{-5 / 2}}{\left(1-(17)^{-1 / 2}\right) 3^{1 / 2}}\right) \approx 1.6008 .
$$

For $p=7, q=13$, we have

$$
|\delta| \leq 12\left(\frac{1}{13}+\frac{7}{13^{2}}+\frac{36}{13^{3}}+\frac{161}{13^{4}}+\frac{659}{13^{5}}+\frac{2(13)^{-3}}{\left(1-(13)^{-1 / 2}\right) 3^{1 / 2}}\right) \approx 1.7144 .
$$

For $p=6, q=10$, we have

$$
|\delta| \leq 9\left(\frac{1}{2 \cdot 5}+\frac{3}{2 \cdot 5^{2}}+\frac{13}{2^{2} 5^{3}}+\frac{6}{5^{4}}+\frac{79}{2^{3} 5^{5}}+\frac{117}{2^{3} 5^{6}}+\frac{10^{-5 / 2}\left(1+10^{-1 / 2}\right)}{9}\right) \approx 1.8014 .
$$

In all three exceptional cases, since $\delta \in \mathbb{Z},|\delta| \leq 1$ also. This completes the proof.

Proof of Theorem 4.1. We prove that $T$ has exactly six neighbors, and then by Theorems 1.1, 1.3 that $T$ is disklike. We divide the proof into four cases and apply Lemma 4.6 throughout. 
Case (i). $f(x)=x^{2}+p x+q, p, q>0$ : as $T+\ell$ is a neighbor of $T$ with $\ell=\gamma v+\delta A v,|\delta| \leq 1$ and $\delta \in \mathbb{Z}, \delta=-1$ or 0 or 1 . We will use the $\delta^{\prime}$ in $\ell^{\prime}=\gamma^{\prime} v+\delta^{\prime} A v$ (Lemma 4.6) to determine $\gamma$. When $\delta=0$, we have $\delta^{\prime}=\gamma-p \delta=\gamma$. But $\left|\delta^{\prime}\right| \leq 1$ (Propositions 4.5, 4.8) and $\gamma \neq 0$ force $\gamma=1$ or -1 . When $\delta=1$, we have $\delta^{\prime}=\gamma-p \delta=\gamma-p$ (by (4.8) ). This implies that $\gamma=p-1$ or $p$ or $p+1$. Similarly, we can show that when $\delta=-1$, we have $\gamma=-(p-1)$ or $-p$ or $-(p+1)$.

We have just shown that there are at most eight points that give potential neighbors of $T$ :

$$
\pm v, \quad \pm(A v+(p-1) v), \quad \pm(A v+p v), \quad \text { and } \quad \pm(A v+(p+1) v) .
$$

We see in Corollary 3.6 that the first three pairs actually give the neighbors. We claim that the last pair does not define any neighbor of $T$. It suffices to consider $A v+(p+1) v$. Suppose $T+A v+(p+1) v$ is a neighbor of $T$. Then we have $\delta^{\prime}=(p+1)-p=1$. By using Lemma 4.6 again on $T+\ell^{\prime}$, we have another neighbor $T+\ell^{\prime \prime}$ of $T$ with $\ell^{\prime \prime}=\gamma^{\prime \prime} v+\delta^{\prime \prime} A v$. Then

$$
\delta^{\prime \prime}=\gamma^{\prime}-p \delta^{\prime}=-q-b_{1}-p \leq-q+(q-1)-p=-p-1<-1,
$$

which contradicts Proposition 4.5. Hence $T$ has exactly six neighbors.

Case (ii). $f(x)=x^{2}-p x+q, p, q>0$. We use the same argument to obtain four pairs, which give the potential neighbors

$$
\pm v, \quad \pm(A v-(p-1) v), \quad \pm(A v-p v), \quad \text { and } \quad \pm(A v-(p+1) v),
$$

and see that only the first three pairs produce neighbors of $T$.

Case (iii). $f(x)=x^{2}+p x-q, p, q>0$. By the same method we get eight potential neighbors from

$$
\pm v, \quad \pm(A v+(p+1) v), \quad \pm(A v+p v), \quad \text { and } \quad \pm(A v+(p-1) v) .
$$

The first three pairs actually give neighbors of $T$ (Corollary 3.6) ). If $T+A v+(p-1) v$ is a neighbor, then $\delta^{\prime}=p-1-p=-1$ and

$$
\delta^{\prime \prime}=\gamma^{\prime}-p \delta^{\prime}=q-b_{1}+p \geq q-(q-1)+p=p+1>1 .
$$

This contradiction shows that $T+A v+(p-1) v$ is not a neighbor; neither is $T-[A v+(p-1) v]$.

Case (iv). $f(x)=x^{2}-p x-q, p, q>0$. We get eight potential neighbors from

$$
\pm v, \quad \pm(A v-(p+1) v), \quad \pm(A v-p v), \quad \text { and } \quad \pm(A v-(p-1) v) .
$$

The same argument shows that only the first three pairs give neighbors of $T$.

\section{REMARKS}

As a graphic illustration of the main theorem (Theorem 1.4), we inspect two tiles generated by different $A$ and $\mathcal{D}$. Let

$$
A=\left[\begin{array}{cc}
0 & 1 \\
-5 & -3
\end{array}\right]
$$

be the companion matrix of $x^{2}+3 x+5$, and let $\mathcal{D}=\left\{(0, i)^{t}: i=0,1,2,3,4\right\}$. As $2 p=6<7=q+2$, by Theorem 1.4 $T_{1}=T(A, \mathcal{D})$ is disklike (see Figure 2).

Let

$$
A=\left[\begin{array}{cc}
0 & 1 \\
-5 & -4
\end{array}\right] \text {, }
$$




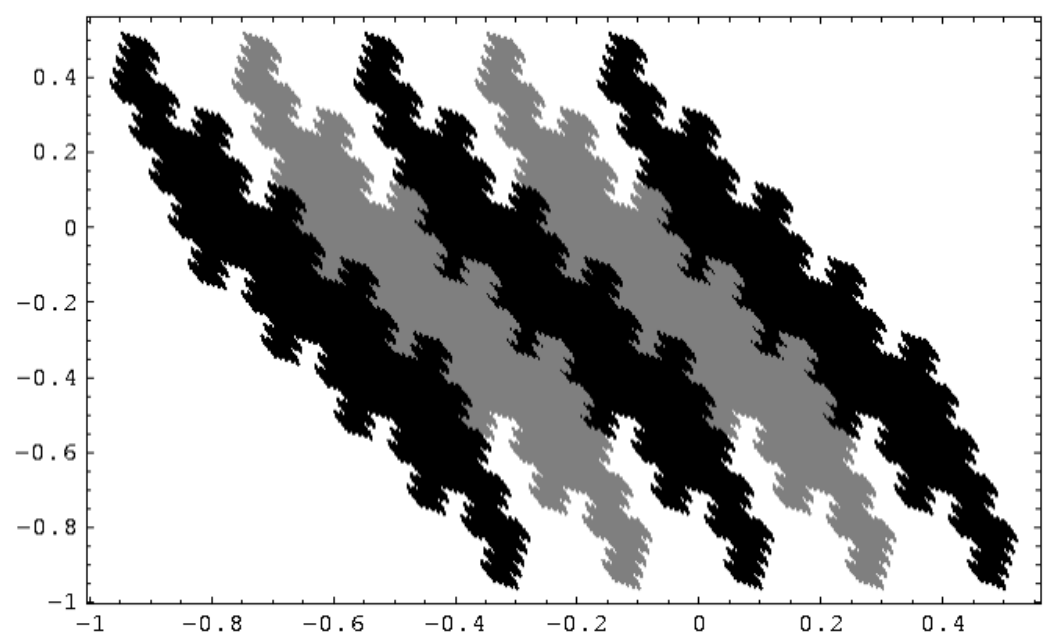

Figure 2. A disklike tile

the companion matrix of $x^{2}+4 x+5$, and let $\mathcal{D}$ be the same as in the previous example. Then $T_{2}=T(A, \mathcal{D})$ is non-disklike as $2 p=8>7=q+2$ (see Figure 3).

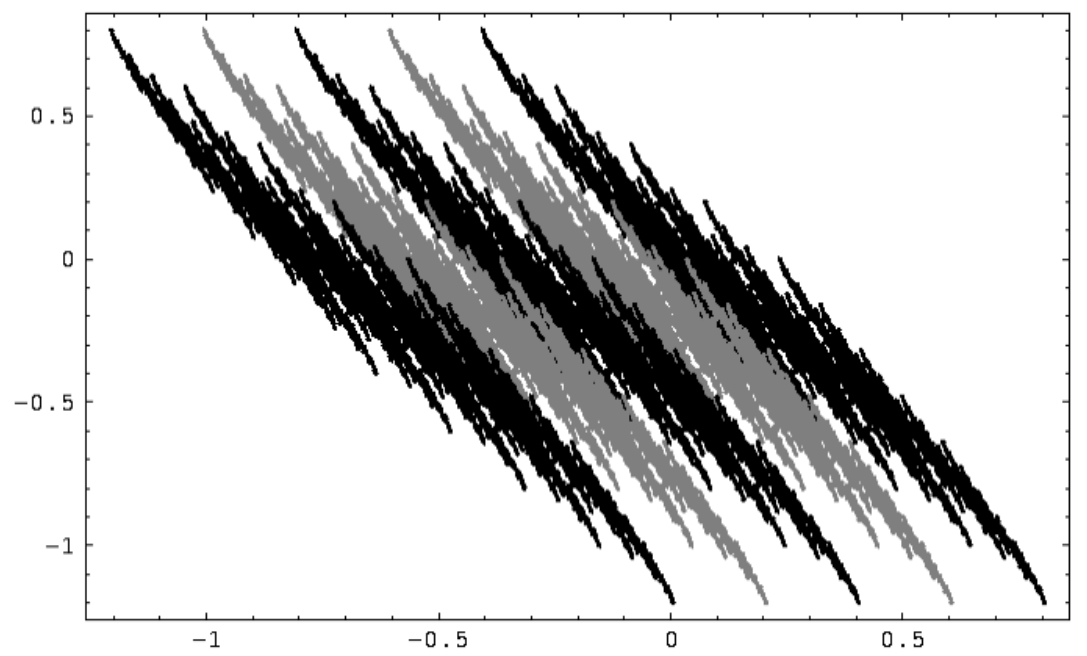

FIGURE 3. A non-disklike tile

It is clear from the picture that $T_{1}$ has six neighbors, but not $T_{2}$ as it can have other neighbors through the slits. Note that $T_{2}$ is connected $\mathrm{KL}$ but $T_{2}^{o}$ is disconnected. In [NT, Ngai and Tang have devised a technique to study tiles of this kind.

It is natural to ask if the non-consecutive collinear digit set will give a disconnected tile. Recently, Tan [T] gave an interesting example that if $A$ is an integral expanding matrix with characteristic polynomial $x^{2}-x-3(|\operatorname{det} A|=3)$ and if $\mathcal{D}=\left\{0, d_{1} v, d_{2} v\right\}$ with $v=[0,1]^{t}$ and $d_{1}<d_{2}$, then $T$ is connected if $\frac{8}{5} d_{1} \leq d_{2} \leq \frac{8}{3} d_{1}$ and it is disconnected if $d_{2}<(\sqrt{13}-1) d_{1} / 2$ or $d_{2}>(\sqrt{13}+5) d_{1} / 2$. 
There is no general knowledge on the connectedness of tiles generated by the nonconsecutive collinear digit sets nor the non-collinear digit sets.

In regard to the extension of disklikeness in higher dimensions, Malone $\mathrm{M}$ ] showed that

Theorem 5.1. In $\mathbb{R}^{n}$, if $T=T(A, \mathcal{D})$ (D may not be collinear) is a parallelepiped, then $A$ is similar to a weighted permutation matrix. Conversely, if $A$ is similar to a weighted permutation matrix, then there exists a digit set $\mathcal{D}$ (may not be collinear) such that $T=T(A, \mathcal{D})$ is a parallelepiped.

There seems to be no known result on disklikeness in higher dimensions for more general matrices.

Finally, we give a discussion on connectedness and an algebraic property introduced in $[\mathrm{KL}$. We say that a polynomial $f(x) \in \mathbb{Z}[x]$ has the height reducing property if there exists $g(x) \in \mathbb{Z}[x]$ such that

$$
g(x) f(x)=x^{k}+a_{k-1} x^{k-1}+\cdots+a_{1} x \pm q
$$

with $\left|a_{i}\right| \leq|q|-1, i=1, \cdots, k-1$. The following was proved in KL.

Theorem 5.2. Let $A \in M_{n}(\mathbb{Z})$ be expanding with $\operatorname{det} A=q$. Suppose the characteristic polynomial of $A$ has the height reducing property. Then for $\mathcal{D}=\{0, v, 2 v$, $\cdots,(|q|-1) v\}$, the tile $T(A, \mathcal{D})$ is connected

The criterion was proved to be valid up to dimension $n=4$ ( $\mathrm{AG}, \mathrm{KLR})$. We conjecture that all expanding polynomials have this property. A weaker form of this was given in $\mathrm{Ga}$ and more supporting evidence was proved in [KLR].

\section{REFERENCES}

[AG] S. Akiyama and N. Gjini, On the connectedness of self-affine attractors, Arch. Math., 82 (2004), 153-163. MR2047669 (2004m:37018)

[AT1] S. Akiyama and J. M. Thuswaldner, A survey on the topological properties of tiles related to number systems, Geom. Dedicata, 109 (2004), 89-105. MR.2113188 (2005h:37035)

[AT2] S. Akiyama and J. M. Thuswaldner, Topological properties of two-dimensional number systems, J. Theor. Nombres Bordeaux, 12 (2000), 69-79. MR:1827838 (2002g:11013)

[AT3] S. Akiyama and J. M. Thuswaldner, On the topological properties of fractal tilings generated by quadratic number systems, Comput. Math. Appl., 49 (2005), no. 7-10, 1439-1485. MR2149493 (2006k:28010)

[B] C. Bandt, Self-similar sets, 5. Integer matrices and fractal tilings of $\mathbb{R}^{n}$, Proc. Amer. Math. Soc., 112 (1991), 549-561. MR1036982 (92d:58093)

[BG] C. Bandt and G. Gelbrich, Classification of self-affine lattice tilings, J. London Math. Soc., 50 (1994), 581-593. MR1299459 (95g:52035)

[BW] C. Bandt and Y. Wang, Disklike self-affine tiles in $\mathbb{R}^{2}$, Discrete Comput. Geom., 26 (2001), 591-601. MR.1863811 (2002h:52028)

[Ba] M. F. Barnsley, Fractals everywhere, second edition (Academic Press, 1993). MR1231795 (94h:58101)

[Ga] A. Garsia, Arithmetic properties of Bernoulli convolutions, Trans. Amer. Math. Soc., 102 (1962), 409-432. MR0137961 (25:1409)

[Gi1] W. J. Gilbert, Complex numbers with three radix representations, Canad. J. Math., 34 (1982), 1335-1348. MR0678674 (85c:11013)

[Gi2] W. J. Gilbert, Complex bases and fractal similarity, Ann. Sci. Math. Québec, 11 (1987), 65-77. MR0912163 (89a:11017)

[HSV] D. Hacon, N. C. Saldanha, and J. J. P. Veerman, Remarks on self-affine tilings, Experiment. Math., 3 (1994), 317-327. MR1341723 (96j:52038)

[H] M. Hata, On the structure of self-similar sets, Japan J. Appl. Math., 2 (1985), 381-414. MR:0839336 (87g:58080) 
[KL] I. Kirat and K. S. Lau, On the connectedness of self-affine tiles. J. London Math. Soc. 62 (2000), 291-304. MR.1772188 (2001i:52027)

[KLR] I. Kirat, K. S. Lau, and H. Rao, Expanding polynomials and connectedness of self-affine tiles, Discrete Comput. Geom., 31 (2004), 275-286. MR2060641 (2005b:52052)

[LW1] J. C. Lagarias and Y. Wang, Integral self-affine tiles in $\mathbb{R}^{n}$. I, Standard and nonstandard digit sets, J. London Math. Soc. 54 (1996), 161-179. MR1395075 (97f:52031)

[LW2] J. C. Lagarias and Y. Wang, Self-affine tiles in $\mathbb{R}^{n}$, Adv. Math., 121 (1996), 21-49. MR.1399601 (97d:52034)

[LW3] J. C. Lagarias and Y. Wang, Integral self-affine tiles in $\mathbb{R}^{n}$. II, Lattice tilings, J. Fourier Anal. and Appl., 3 (1997), 83-102. MR1428817 (98b:52026)

[L] K.-S. Leung, The radix expansions and the disklikeness of self-affine tiles,. Ph.D. thesis, The Chinese University of Hong Kong, 2004.

[LAT] J. Luo, S. Akiyama, and J. M. Thuswaldner, On the boundary connectedness of connected tiles, Math. Proc. Cambridge Phil. Soc., 137 (2004), 397-410. MR2092067 (2005g:37032)

[LRT] J. Luo, H. Rao and B. Tan, Topological structure of self-similar sets, Fractals, 10 (2002), 223-227. MR.1910665 (2003d:28014)

[M] D. Malone, Solutions to dilation equations, Ph.D. thesis, University of Dublin, 2000.

[NT] S. M. Ngai and T. M. Tang. A technique in the topology of connected self-similar tiles, Fractals, 12, (2004), no. 4, 389-403. MR.2109984 (2006b:52018)

[O] A. M. Odlyzko. Nonnegative digit sets in positional number systems, Proc. London Math. Soc., 37 (1978), 213-229. MR0507604 (80m:10004)

[SK] H. J. Song and B. S. Kang. Disclike lattice reptiles induced by exact polyominoes, Fractals, 7 (1999), 9-22. MR.1687038 (2000a:52034)

[T] B. Tan, Private communication.

Department of Mathematics, Science, Social Sciences and Technology, The Hong Kong Institute of Education, Tai Po, Hong Kong

E-mail address: ksleung@ied.edu.hk

Department of Mathematics, The Chinese University of Hong Kong, Shatin, Hong KONG

E-mail address: kslau@math.cuhk.edu.hk 